\title{
Innovative Technologies for Reducing Structural Vibrations due to Natural Events and Human Activities
}

\author{
Nicola Caterino, ${ }^{1}$ Christos T. Georgakis, ${ }^{2}$ Ningsu Luo, ${ }^{3}$ and Julian Londono Monsalve ${ }^{4}$ \\ ${ }^{1}$ Department of Engineering, University of Naples Parthenope, 80143 Naples, Italy \\ ${ }^{2}$ Department of Engineering, Aarhus University, 8000 Aarhus C, Denmark \\ ${ }^{3}$ Department of Electrical Engineering, Electronics and Automatic Control, University of Girona, 17071 Girona, Spain \\ ${ }^{4}$ College of Engineering, Mathematics and Physical Sciences, University of Exeter, Exeter, Devon, UK \\ Correspondence should be addressed to Nicola Caterino; nicola.caterino@uniparthenope.it
}

Received 1 September 2016; Accepted 4 September 2016

Copyright (c) 2016 Nicola Caterino et al. This is an open access article distributed under the Creative Commons Attribution License, which permits unrestricted use, distribution, and reproduction in any medium, provided the original work is properly cited.

The need for effective structural control systems able to improve the performance of new and existing structures against service and extreme loads is ever growing. Indeed, over the last few decades the reduction of unwanted dynamics became a field at which intense research effort has been devoted and significant developments have been achieved by major research groups worldwide. This special issue aims at networking novel researches concerning complex and edge-technology systems and methodologies addressed to vibration control of civil structures subjected to natural actions as well as to dynamic loading induced by human activities.

M. Berardengo et al. deal with mono-modal vibration attenuation using piezoelectric transducers shunted to impedances consisting of an inductance and a resistance in series. They analyze the behaviour of mistuned electromechanical systems, demonstrating that a linear relationship between the attenuation and the logarithm of the effective coupling coefficient exists not only when a perfect tuning is given, but also when there is mistuning on either the electrical eigenfrequency or damping. Furthermore, the authors show how the loss of attenuation mainly depends on only one bias if the electrical damping is overestimated and describe how the effects of the two bias types combine with each other when the damping is underestimated. An approximated model to simulate the behaviour of mistuned shunt systems has been proposed, numerically validated via Monte Carlo simulations and then experimentally tested with two sample structures.
Earthquakes may lead to damage and collapse of colliding structures in the case of insufficient gap between adjacent structures. Q. Xue et al. propose an updated pounding force analysis method based on viscoelasticity of materials. Comparisons among proposed pounding force model and other traditional models are analyzed. Moreover, the proposed model is applied to simulate the pounding tuned mass damper (TMD) device so as to verify the validity of the model. The proposed method expands traditional Kelvin viscoelastic model to 3-parameter linear solid viscoelastic model.

Light weight, low stiffness structures potentially may suffer from wind-induced vibrations and fatigue failure. Gumus and Ertas perform theoretical analysis for a novel free-pendulum device to be adopted as a passive vibration absorber, suitable for the mitigation of large amplitude windinduced vibrations in traffic signal structures.

J. Marra et al. deal with the development and practical implementation of a hybrid (passive-active/adaptive) vibration control system over a metallic beam excited by a broadband signal and under variable temperature. Temperature variations may affect considerably the performance of the passive control system, a viscoelastic dynamic vibration absorber. The authors show that coupling the passive device with an active-adaptive vibrations control system, based on a feedforward approach with the use of the FXLMS algorithm, leads to compensating the neutralizer loss of performance, even improving the attenuation of vibrations levels. 
A magnetorheological damper with energy harvesting ability is proposed by G. Hu et al. It exploits the electromagnetic induction principle. The energy harvesting part is made of a magnet array and coils moving vertically. When the external excitation is applied, the kinetic energy is converted into electrical energy. Finite element modelling and experimental tests show good capability of the variable damper in changing mechanical behaviour according to the intensity of the feeding current, and considerable voltage output harvested with the proposed technique.

$\mathrm{X}$. Li et al. deal with mega-sub-isolation system addressed to mitigate structural vibrations. The working mechanism of the mega-sub-isolation system is obtained by systematically investigating its dynamic characteristics corresponding to various structural parameters. Considering the number and location of the isolated substructures, an optimization procedure to design the isolation system is proposed, based on a genetic algorithm where base shear is assumed as objective function. The control performance of such megasub-isolation system is also investigated from the perspective of energy.

M.-H. Shih and W.-P. Sung improve the seismic proofing capability of an accumulated semiactive hydraulic damper, making it able to behave like an active interaction control device by means of synchronous control and predictive control methods. A full scale shaking table test is done to verify the performance of the proposed control system, leading to show that, using suitable stiffeners for the auxiliary structure at each floor, with synchronous control and predictive control, the technique guarantees high reliability and practicability for seismic proofing of buildings under excitation by external forces.

\section{Acknowledgments}

The guest editors would like to thank the authors for their contributions to the special issue and all the reviewers for their constructive reviews.

Nicola Caterino

Christos T. Georgakis

Ningsu Luo

Julian Londono Monsalve 


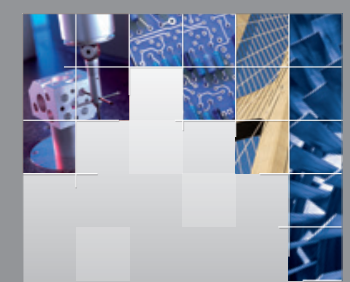

\section{Enfincering}
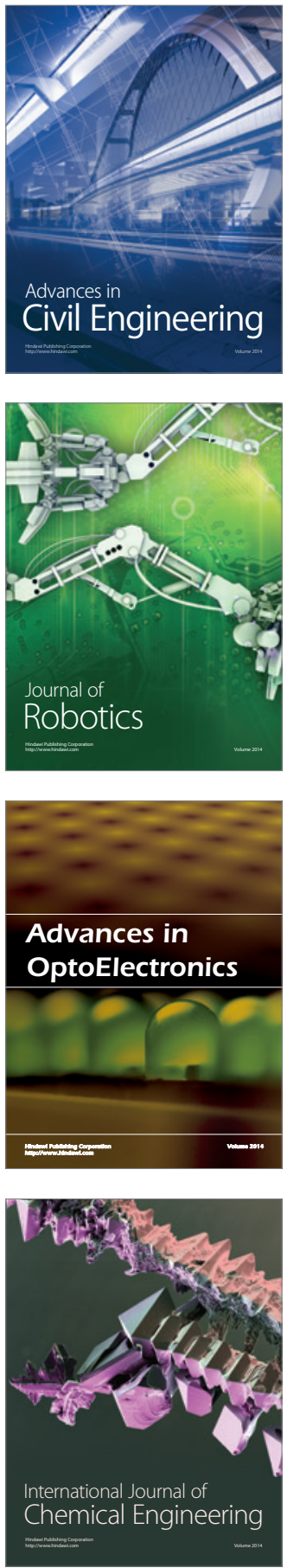

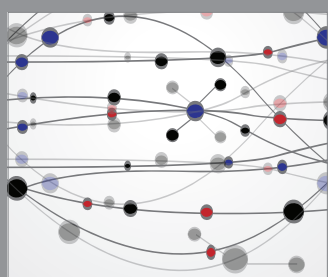

The Scientific World Journal

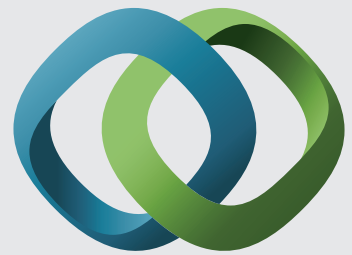

\section{Hindawi}

Submit your manuscripts at

http://www.hindawi.com
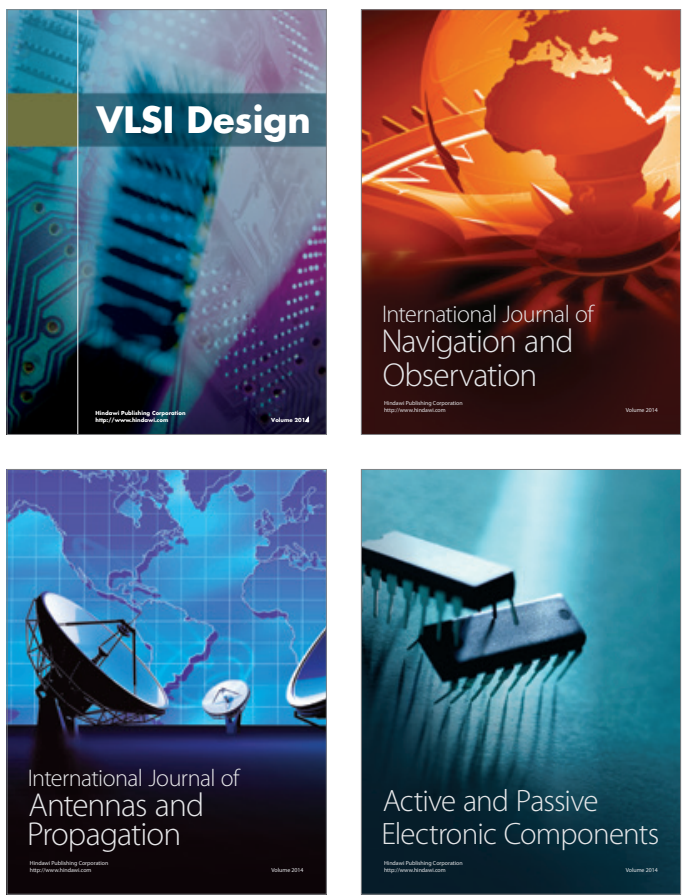
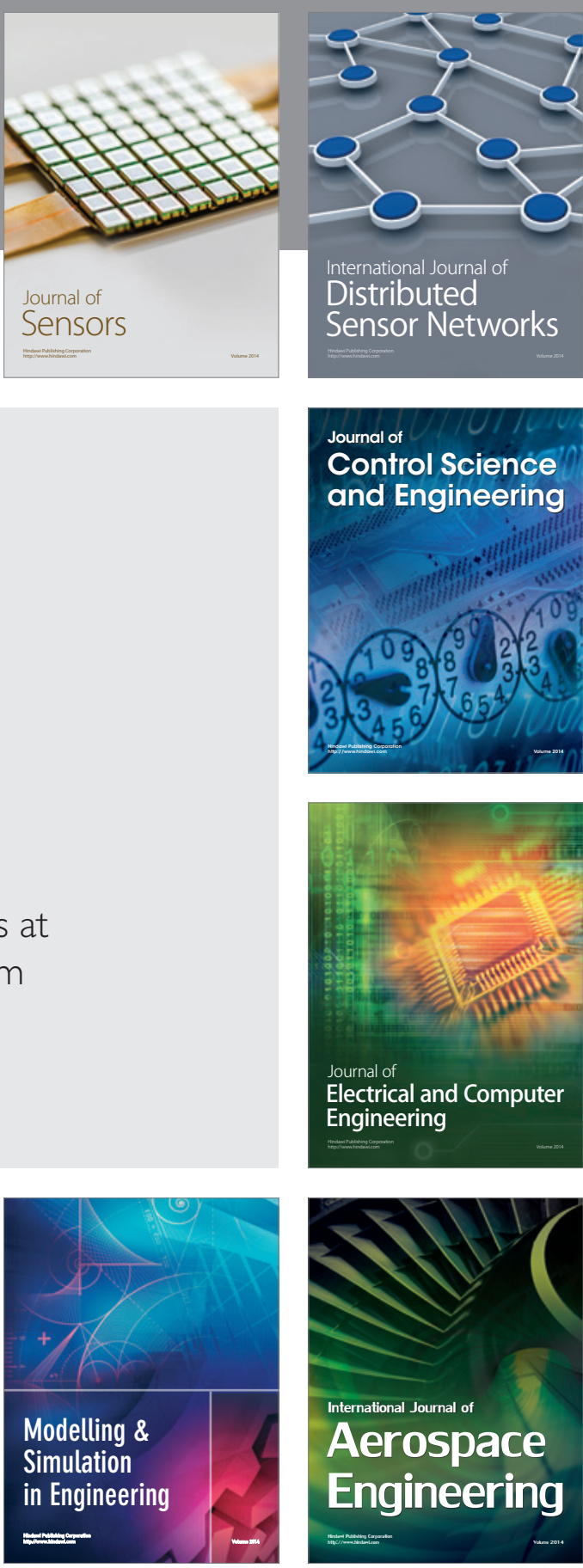

International Journal of

Distributed

Sensor Networks

Journal of

Control Science

and Engineering
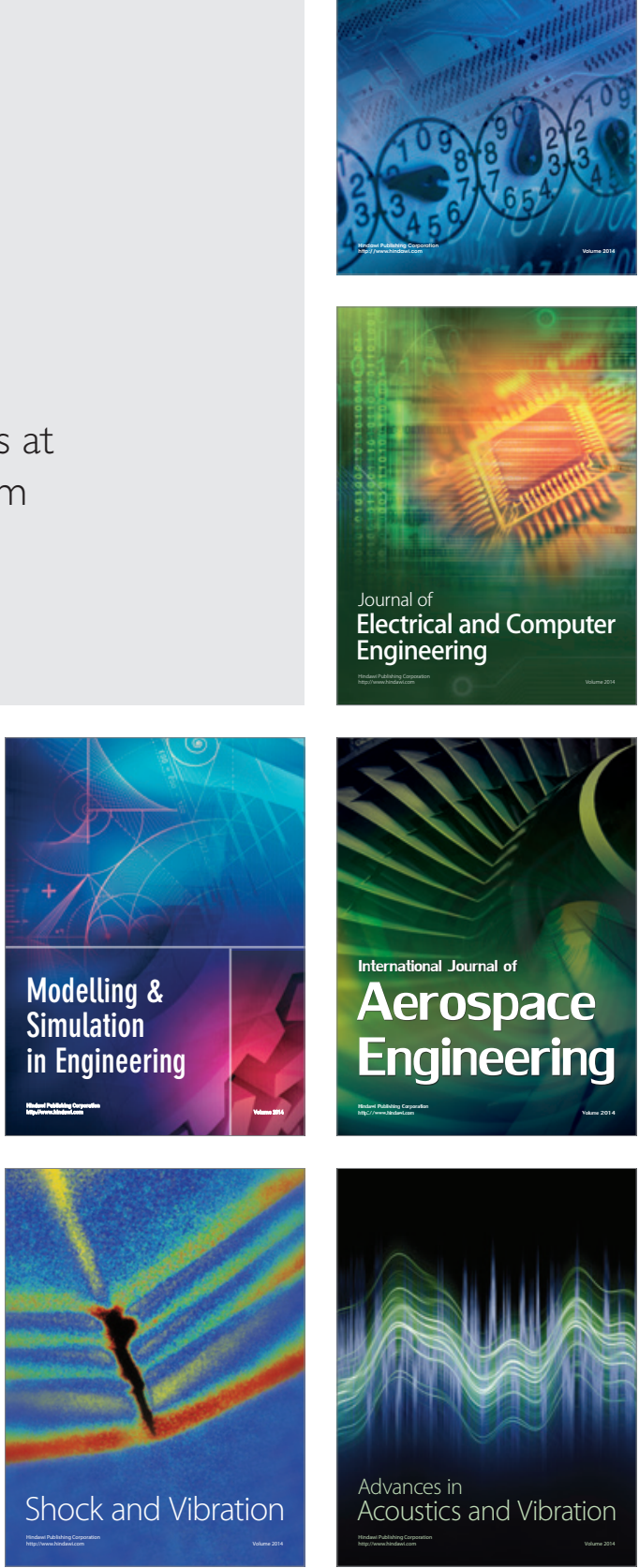